\title{
Investigating the origin of strangeness enhancement in small systems through multi-differential analyses
}

\author{
Francesca Ercolessi $^{1, *}$ (for the ALICE Collaboration) \\ ${ }^{1}$ University and INFN, Bologna, Italy
}

\begin{abstract}
The main goal of the ALICE experiment is to study the physics of strongly interacting matter, including the properties of the quark-gluon plasma (QGP). The relative production of strange hadrons with respect to non-strange hadrons in heavy-ion collisions was historically considered as one of the signatures of QGP formation. However, measurements at the LHC in pp and $\mathrm{p}-\mathrm{Pb}$ collisions have shown similar features to those observed in $\mathrm{Pb}-\mathrm{Pb}$, measuring an increase in the production of strange hadrons relative to pions with the chargedparticle multiplicity in the event. In order to better understand the role of the event multiplicity to the observed enhancement in pp, two new complementary analyses have been performed. The first exploits the angular correlation between strange and high- $p_{\mathrm{T}}$ hadrons in the event to classify in-jet and out-of-jet strangeness production. The second uses the concept of the effective energy available in the event for particle production which is estimated by an anticorrelation with the energy deposited in ALICE's Zero Degree Calorimeters. The results suggest that strangeness enhancement emerges from the growth of the underlying event and is not connected to initial state properties.
\end{abstract}

\section{Introduction}

Strange particle production in heavy-ion collisions has proved to be an important observable to investigate the strongly interacting medium created in the collision. Strangeness enhancement was one of the first proposed signatures of quark-gluon plasma (QGP) formation [1], which is expected to lead to an increased production of strange hadrons relative to pp interactions. The enhancement was predicted to follow a hierarchy related to the strangeness content of the particle, being stronger for multi-strange hadrons. This phenomenon was observed for the first time at SPS [2], then at RHIC [3] and later at the LHC [4] at increasing collision energies. The ALICE experiment has also studied strangeness production in small collision systems, such as $\mathrm{p}-\mathrm{Pb}$ and $\mathrm{pp}$, by measuring the ratio of strange particle yields to pion yields as a function of the charged-particle multiplicity produced in the event. This ratio is found to increase across multiplicities, evolving smoothly within different collision systems and energies. The results can be compared to various model predictions, from a statistical hadronization description using the canonical suppression approach [5], to rope hadronization models including colour reconnection effects [7], to two-component (core-corona) models [6]. However, the mechanisms responsible for this intriguing phenomenon observed in small systems are still unclear and further studies are needed.

\footnotetext{
*e-mail: francesca.ercolessi@cern.ch
} 


\section{Results}

The ALICE experiment identifies $K_{\mathrm{S}}^{0}, \Lambda, \Xi$ and $\Omega$ baryons by reconstructing their weak decay daughter tracks in the central pseudorapidity region. The main subdetectors used for particle identification (PID) and tracking include the six-layer high resolution Inner Tracking System (ITS) and the large volume Time Projection Chamber (TPC). Two forward detectors placed on both sides of the ALICE interaction point, the V0 and the Zero Degree Calorimeters (ZDC), are used to classify events in multiplicity and energy event classes, respectively. A detailed description of the ALICE apparatus and its performance can be found in [8]. The two analyses presented in these proceedings exploit multi-differential approaches to explore strangeness enhancement in pp.

The processes involved in particle production in $\mathrm{pp}$ collisions can be classified into hard and soft ones, depending on the momentum transfer. The relative contribution of hard and soft processes to strangeness production in $\mathrm{pp}$ is still not fully understood and can be studied through techniques involving two-particle correlations. In the first analysis ALICE exploits the angular correlation method to separate $\mathrm{K}_{\mathrm{S}}^{0}$ and $\Xi$ hadrons produced in jets (hard processes) from those produced out-of-jets (soft processes). Particles produced in the near-side jet region are characterised by a small angular separation from the leading particle of the jet, which is identified as the particle with the highest transverse momentum in the collision with $p_{\mathrm{T}}>3 \mathrm{GeV} / c$. The angular correlation distribution of the jet trigger particle and the associated strange hadron is studied in the $(\Delta \eta, \Delta \phi)$ plane, which is divided into a near-side-jet region, an out-of-jet region, and a full inclusive region. The near-side-jet contribution is obtained subtracting the full and out-of-jet in the $(\Delta \eta, \Delta \phi)$ corresponding region. The $\Xi$ yields per trigger particle and per unit of $\Delta \eta \Delta \phi$ are displayed in Fig. 1 as a function of the charged-particle multiplicity produced at midrapidity.

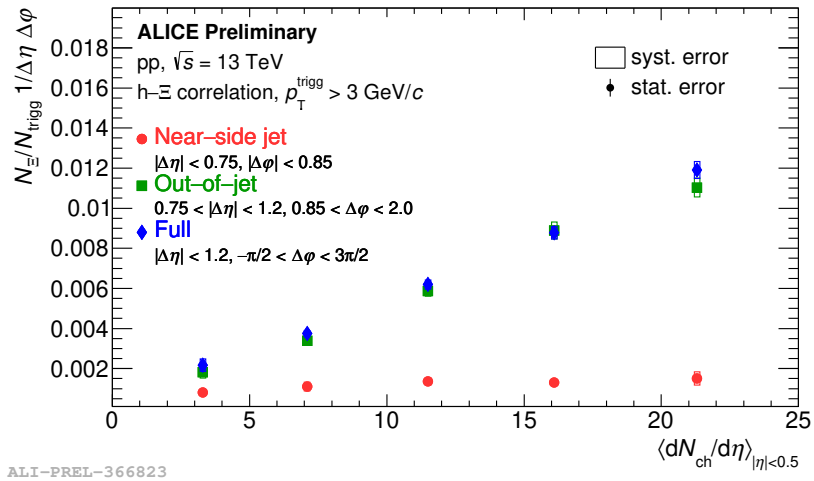

Figure 1. $\Xi$ yields per trigger particle and per unit of $\Delta \eta \Delta \phi$ as a function of the charged-particle multiplicity produced at midrapidity.

The full and out-of-jet yields increase with multiplicity, while the near-side-jet yields show a very mild to no dependence on particle production at midrapidity. Similar results are obtained for $\mathrm{K}_{\mathrm{S}}^{0}$ yields. These results suggest that out-of-jet (soft) processes are the dominant contribution to strange particle production in pp collisions.

The multiplicity distribution of charged hadrons is an important characteristic of the hadronic final state of any collision. But it also reflects the initial dynamics of the interaction, 
being strongly correlated with the energy effectively available for particle production in its initial stages (effective energy). In pp collisions the effective energy is reduced with respect to the full centre-of-mass energy due to the leading effect, which consists of a high probability to emit forward baryons with large longitudinal momenta along the direction of the incident beams. More details can be found in [9] and references therein. The effective energy can be estimated by means of ALICE's ZDC by detecting the energy deposits of the forward leading baryons in each event hemisphere: $E_{\text {effective }}=\sqrt{s}-E_{\text {leading. In the second }}$ analysis the events are classified in effective energy and multiplicity percentile classes, using the information from the ZDC and V0 detectors, respectively, in order to disentangle initial and final state effects on strange hadron production. The correlation between effective energy and particle multiplicity in pp is studied using the PYTHIA 8 event generator in Fig. 2 (left), which shows that events selected using a V0 or ZDC based estimator alone are sensitive to both initial and final state. Fig. 2 (right) shows the ratio of $\Xi$ yields to the charged-particle multiplicity in the event (self-normalized to INEL $>0^{1}$ ) as a function of particle production at midrapidity selected using V0 or ZDC. $\Xi$ hadron production is found to increase with multiplicity following a similar trend, independent of the estimator used.
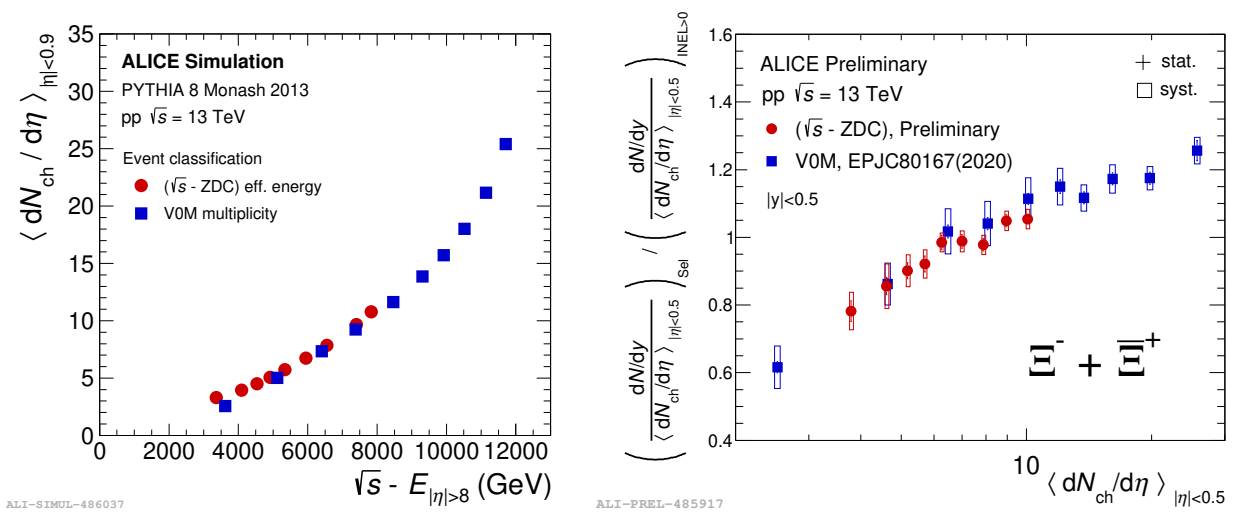

Figure 2. (left) Multiplicity and effective energy correlation in PYTHIA 8 using V0 and ZDC event classes. (right) Ratio of $\Xi$ yields to the charged-particle multiplicity (self-normalized to INEL $>0$ ) as a function of particle production at midrapidity, using V0 and ZDC standalone selections.

In order to separate initial and final state contributions to strange particle production, V0 and ZDC combined classes are exploited. Their discriminating power in terms of effective energy and multiplicity is studied in Fig. 3 (left) via the PYTHIA 8 event generator. Events are selected using ZDC percentile selections, fixing the multiplicity through the V0 estimator in a high and low range (red points), and vice-versa using V0 percentile selections, fixing the effective energy through the ZDC estimator to a high and low range (blue points). Fig. 3 (right) shows the ratio of $\Xi$ yields to the charged-particle multiplicity (self-normalized to INEL $>0$ ) selected using ZDC event classes, fixing the multiplicity through the V0 estimator. The points are distributed in a flat trend showing no dependence on the energy percentile estimator. The different V0 selection is responsible for the enhancement observed between the two sets of points. The results suggest that the effective energy does not play a significant role in the strangeness enhancement observed in pp collisions, which is found to be driven by the final state multiplicity.

\footnotetext{
${ }^{1}$ INEL $>0$ is a conventional event class which contains events with at least one charged particle in $|\eta|<1$.
} 

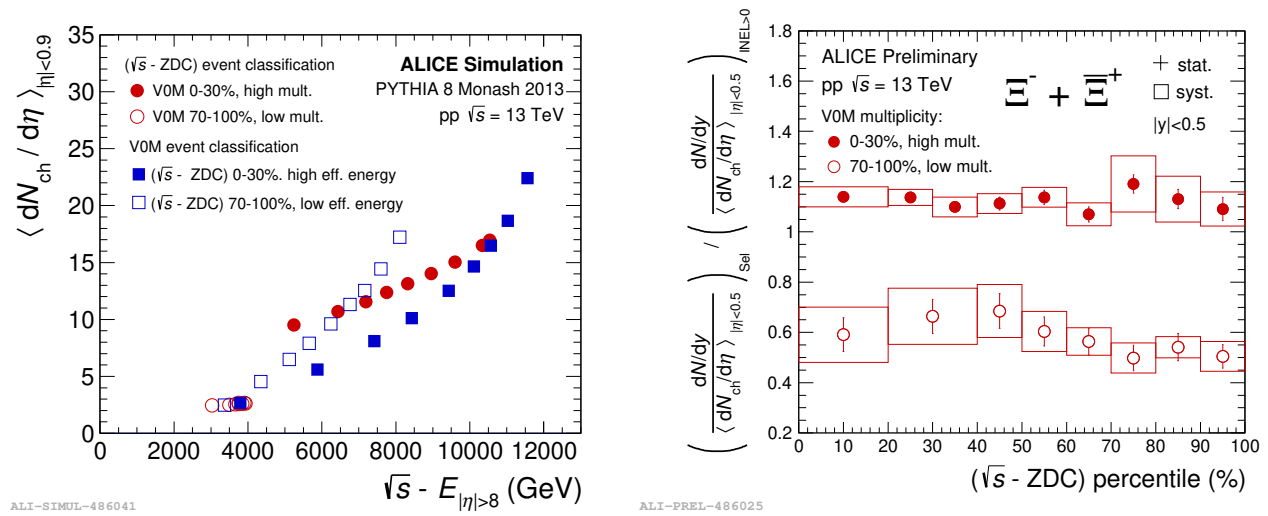

Figure 3. (left) Multiplicity and effective energy correlation in PYTHIA 8 using V0-ZDC combined classes. (right) Ratio of $\Xi$ yields to the charged-particle multiplicity (self-normalized to INEL $>0$ ) extracted using V0-ZDC combined selections. Events are selected using ZDC event classes, fixing the multiplicity through the V0 estimator.

\section{Conclusions}

The ALICE Collaboration has provided a comprehensive set of results on the production of strange hadrons in pp collisions, however, the mechanisms responsible of the strangeness enhancement with multiplicity observed in small systems are still not fully understood. These preliminary results exploit a multi-differential approach to disentangle initial and final state effects on strange hadron production, suggesting a strong connection with the final particle multiplicity and no significant role of the initial effective energy. Moreover, soft out-of-jet processes are found to be the dominant contribution, with respect to in-jet hard processes, to strangeness production.

\section{References}

[1] J. Rafelski, B. Müller, Phys. Rev. Lett. 48, 1066-1069 (1982).

[2] The WA97 Collaboration, Physics Letters B 449, 401-406 (1999).

[3] Agakishiev, G. et al. (STAR Collaboration) Phys. Rev. Lett. 108, 072301 (2012).

[4] Abelev, B. et al. (ALICE Collaboration) Phys. Lett. B 728, 216 (2014).

[5] Vovchenko, V. et al., Phys. Rev. C 100, 054906 (2019).

[6] Kanakubo, Y. et al., Phys. Rev. C 101, 024912 (2020).

[7] Nayak, R. et al., Phys. Rev. D 100, 074023 (2019).

[8] Aamodt, K. et al., (ALICE Collaboration) JINST 3, S08002 (2008).

[9] Akindinov, A., et al. Eur. Phys. J. C 50, 341-352 (2007). 